\title{
Localized rotating convection with no-slip boundary conditions
}

\author{
Cédric Beaume, ${ }^{1, \text { a) }}$ Hsien-Ching Kao, ${ }^{1, b)}$ Edgar Knobloch, ${ }^{1, c)}$ \\ and Alain Bergeon ${ }^{2, d)}$ \\ ${ }^{1}$ Department of Physics, University of California, Berkeley, California 94720, USA \\ ${ }^{2}$ Université de Toulouse, INPT, UPS, IMFT (Institut de Mécanique des Fluides de Toulouse), \\ Allée Camille Soula, F-31400 Toulouse, France and CNRS, IMFT, F-31400 Toulouse, France
}

(Received 29 July 2013; accepted 22 November 2013; published online 20 December 2013)

Localized patches of stationary convection embedded in a background conduction state are called convectons. Multiple states of this type have recently been found in two-dimensional Boussinesq convection in a horizontal fluid layer with stress-free boundary conditions at top and bottom, and rotating about the vertical. The convectons differ in their lengths and in the strength of the self-generated shear within which they are embedded, and exhibit slanted snaking. We use homotopic continuation of the boundary conditions to show that similar structures exist in the presence of no-slip boundary conditions at the top and bottom of the layer and show that such structures exhibit standard snaking. The homotopic continuation allows us to study the transformation from slanted snaking characteristic of systems with a conserved quantity, here the zonal momentum, to standard snaking characteristic of systems with no conserved quantity. 2013 AIP Publishing LLC. [http://dx.doi.org/10.1063/1.4843155]

\section{INTRODUCTION}

The competition between destabilizing thermal forcing and a stabilizing constraint results in rich dynamics in a variety of applications including doubly diffusive convection, ${ }^{1}$ magnetoconvection, ${ }^{2}$ and rotating convection. ${ }^{3}$ A review of the instability mechanisms in these systems can be found in Ref. 4. Recent work on these systems has identified the presence of spatially localized convection in the form of structures that have been called convectons ${ }^{5-10}$ and the presence of such structures has been related to the phenomenon of homoclinic snaking that has been extensively studied in the context of model systems such as the Swift-Hohenberg equation. ${ }^{11-14}$ These new states consist of a finite number of rolls embedded in a background conduction state and are located in the so-called snaking or pinning region in parameter space. Within this region the convectons lie on a pair of intertwined branches: in systems with midplane symmetry these consist of states of odd and even parity; when this symmetry is absent the branches consist of even parity states with either downflow or upflow in their center. In both cases, the snaking branches are interconnected by branches of asymmetric convectons, forming a snakes-and-ladders structure. ${ }^{11,12}$ In the following, we refer to this structure as standard snaking. It is also known that the form of the resulting bifurcation diagram is significantly altered in the presence of a conserved quantity. ${ }^{15,16}$ This is because the localized structures typically expel the conserved quantity thereby raising its magnitude in the region outside. This in turn modifies the background state and leads to so-called slanted snaking. ${ }^{15-18}$ The presence of slanted snaking implies that localized states are present over a much wider interval in parameter space than is the case with standard snaking.

\footnotetext{
a) ced.beaume@gmail.com

b) sp000088@gmail.com

c) knobloch@ berkeley.edu

d) alain.bergeon@imft.fr
} 
Slanted snaking is a consequence of a conserved quantity, such as imposed magnetic flux in magnetoconvection ${ }^{19}$ or fixed zonal momentum in rotating convection with stress-free boundary conditions at top and bottom, ${ }^{18}$ and is a finite size effect - in an unbounded domain the conserved quantity exerts no effect and the system reverts to standard snaking. ${ }^{17}$ In this paper, we seek to understand the transformation from slanted to standard snaking as the conservation of the conserved quantity is progressively broken and the associated changes in the width of the parameter interval within which convectons may be found. For this purpose, we select convection in a rotating layer where slanted snaking has recently been studied ${ }^{18}$ and employ homotopic continuation of the boundary conditions from stress-free boundary conditions for which slanted snaking is present to no-slip boundary conditions. The procedure allows us to (a) show that convectons in a rotating layer are present even in the presence of the more realistic no-slip boundary conditions, (b) determine the width of the Rayleigh number interval within which they are found, (c) track the morphology changes in the associated bifurcation diagram as the boundary conditions are deformed, and (d) quantify the influence of the conserved zonal momentum on the behavior of this system. We emphasize that homotopic continuation of the boundary conditions is an effective method for finding localized structures in situations where one has no a priori idea of their location, or even if such solutions exist in the first place.

For this purpose, we focus on convection in a Boussinesq fluid layer rotating about a vertical axis at a constant angular velocity $\Omega \hat{\mathbf{z}}$, where $\hat{\mathbf{z}}$ is the unit vector in the vertical direction. The lower (resp. upper) boundary is maintained at a fixed temperature $\Theta_{r}+\Delta \Theta$ (resp. $\Theta_{r}$ ), where $\Theta_{r}$ is a reference temperature and $\Delta \Theta>0$. This physical configuration has been studied for the wealth of instabilities it exhibits ${ }^{3,20,21}$ and for the small amplitude but complex dynamics that result. ${ }^{22-24} \mathrm{We}$ restrict attention to two-dimensional convection described by the dimensionless equations, written in the rotating frame,

$$
\begin{aligned}
\sigma^{-1}\left[\nabla^{2} \psi_{t}+J\left(\psi, \nabla^{2} \psi\right)\right] & =\operatorname{Ra} \theta_{x}-\operatorname{Ta} v_{z}+\nabla^{4} \psi, \\
\theta_{t}+J(\psi, \theta) & =\psi_{x}+\nabla^{2} \theta, \\
\sigma^{-1}\left[v_{t}+J(\psi, v)\right] & =\text { Ta } \psi_{z}+\nabla^{2} v .
\end{aligned}
$$

Here $J(f, g) \equiv f_{x} g_{z}-f_{z} g_{x}$ and $\psi(x, z, t)$ is a streamfunction defined such that the fluid velocity $\mathbf{u}=\left(-\psi_{z}, v, \psi_{x}\right)$ in $(x, y, z)$ coordinates. In the following, we refer to $\psi(x, z, t)$ as the poloidal streamfunction and to $v(x, z, t)$ as the zonal velocity. The quantity $\theta(x, z, t)$ measures the flowinduced departure of the temperature from the conduction profile and satisfies $\theta=0$ at $z= \pm 1 / 2$. In writing these equations, we used the height $h$ of the layer as the unit of length, the thermal diffusion time $h^{2} / \kappa$ (where $\kappa$ is the thermal diffusivity) as the unit of time, and the imposed temperature difference $\Delta \Theta$ as the unit of temperature. The resulting system is specified by the Prandtl number $\sigma$, the Rayleigh number Ra, and the Taylor number Ta, where

$$
\sigma=\frac{\nu}{\kappa}, \quad \mathrm{Ra}=\frac{g \alpha \Delta \Theta h^{3}}{\kappa \nu}, \quad \mathrm{Ta}=\frac{2 \Omega h^{2}}{\nu} .
$$

Here $v$ is the kinematic viscosity, $g$ is the gravitational acceleration, and $\alpha$ is the thermal expansion coefficient. Implicit in the above formulation is the assumption that the Froude number $\Gamma h \Omega^{2} / g$ $\ll 1$, where $\Gamma$ is the aspect ratio of the domain. This assumption allows us to ignore the effects of the centrifugal force. We likewise adopt periodic boundary conditions in the horizontal to avoid complications arising from realistic lateral boundaries when the length of the convectons we compute becomes comparable to the lateral extent of the domain.

In the following, we present bifurcation diagrams that are computed using numerical continuation ${ }^{25}$ based on the use of a time-stepper, ${ }^{26,27}$ as implemented in Refs. 8, 18, and 28. The system described by Eqs. (1)-(3) is discretized in time using a first-order Adams-Bashforth scheme. The domain $\mathcal{D} \equiv[-\Gamma / 2, \Gamma / 2] \times[-1 / 2,1 / 2]$ is decomposed into $N=40$ equal rectangular spectral elements, each discretized using Gauss-Lobatto-Legendre points in the two directions. Unless otherwise stated, we compute stationary solutions in a domain with $\Gamma=20 \lambda_{c}$, where $\lambda_{c}$ 
is the critical wavelength at the onset of stationary convection, using 15 points in the horizontal and 13 points in the vertical for each element. The method is complemented here by an influence matrix technique in order to compute solutions of the incompressible Navier-Stokes equations in the vorticity-streamfunction formulation with different boundary conditions. ${ }^{29}$

The paper is organized as follows. In Sec. II, we summarize the properties of localized states with stress-free boundary conditions and $\mathrm{Ta}=60, \sigma=0.1$, followed in Sec. III by the corresponding results for no-slip boundary conditions. Section IV describes the evolution of the snaking structure as the conservation law is progressively broken. The paper concludes in Sec. V with a discussion of the results.

\section{STRESS-FREE CONVECTONS}

In previous studies, ${ }^{18,30,31}$ stress-free, fixed temperature boundary conditions have been applied at the upper and lower boundaries, i.e.,

$$
\psi=\psi_{z z}=v_{z}=0
$$

at $z= \pm 1 / 2$, together with periodic boundary conditions applied in the horizontal direction at $x=$ $\pm \Gamma / 2$. As formulated, the system possesses $O(2) \times Z_{2}$ symmetry, reflecting its equivariance under horizontal translations $T_{\ell}$, i.e., $x \rightarrow x+\ell$ with the other variables unchanged, as well as the two reflections

$$
\begin{aligned}
& R_{x}:(x, z) \rightarrow(-x, z), \quad(\psi, \theta, v) \rightarrow(-\psi, \theta,-v), \\
& R_{z}:(x, z) \rightarrow(x,-z), \quad(\psi, \theta, v) \rightarrow(-\psi,-\theta, v) .
\end{aligned}
$$

The group $O(2)$ is generated by $T_{\ell}$ and $R_{x}$ while $Z_{2}$ is generated by $R_{z}$. Note that solutions preserving the $R_{z}$ symmetry have to have an even number of rolls in the vertical. This symmetry is therefore always broken by solutions bifurcating at the lowest values of the Rayleigh number. The resulting solutions are therefore translation-invariant and either even (invariant with respect to $R_{x}$ ) or odd (invariant with respect to the point symmetry $R_{c}=R_{x} \circ R_{z}$ ). The symmetries $R_{x}$ and $R_{z}$ indicate that clockwise and counterclockwise rolls contribute equally to the generation of zonal shear.

The zonal velocity $v$ is only defined up to a constant, i.e., it is a phase-like variable. One can show by integrating Eq. (3) over the domain and applying the boundary conditions (5) that its spatial average remains constant in time, i.e.,

$$
\frac{d}{d t} \bar{V}=0
$$

where $\bar{V}$ represents the average of $v$ over $\mathcal{D}$. In the following, we fix the constant of integration to be zero so that the zonal velocity has zero average. The conservation law (8) or equivalently the global constraint $\bar{V}=0$ has important consequences as we shall see.

The system admits the conduction solution $\psi=\theta=v=0$ at all values of Ra. Instability of this state is responsible for the occurrence of convection as predicted by linear stability analysis. ${ }^{4}$ The critical Rayleigh number, defined as the threshold for steady convection, is given by $\operatorname{Ra}_{c}=3 p_{c}^{2}$, where $p_{c} \equiv k_{c}^{2}+\pi^{2}$, and the corresponding critical wavenumber $k_{c}$ satisfies

$$
\mathrm{Ta}^{2} \pi^{2}=p^{2}\left(2 k_{c}^{2}-\pi^{2}\right)
$$

Figure 1 shows the bifurcation diagram obtained for $\mathrm{Ta}=60$ and $\sigma=0.1\left(k_{c} \approx 4.7121\right.$, hence $\Gamma \approx 26.6682$ ). The results are shown in terms of the in-plane (i.e., poloidal) kinetic energy density

$$
\mathcal{E}=\frac{1}{2 \Gamma} \int_{\mathcal{D}}\left[\left(\partial_{x} \psi\right)^{2}+\left(\partial_{z} \psi\right)^{2}\right] d x d z
$$

as the Rayleigh number is varied. With this choice of parameter values the first stationary bifurcation occurs at $\mathrm{Ra}_{c} \approx 3086$ and gives rise to a periodic convection state labeled $P_{20}$. The solutions on this branch are stationary and consist of 20 pairs of counter-rotating rolls in the rotating frame. Each solution breaks the translation invariance of the trivial solution but preserves the symmetry $R_{x}$ (with 

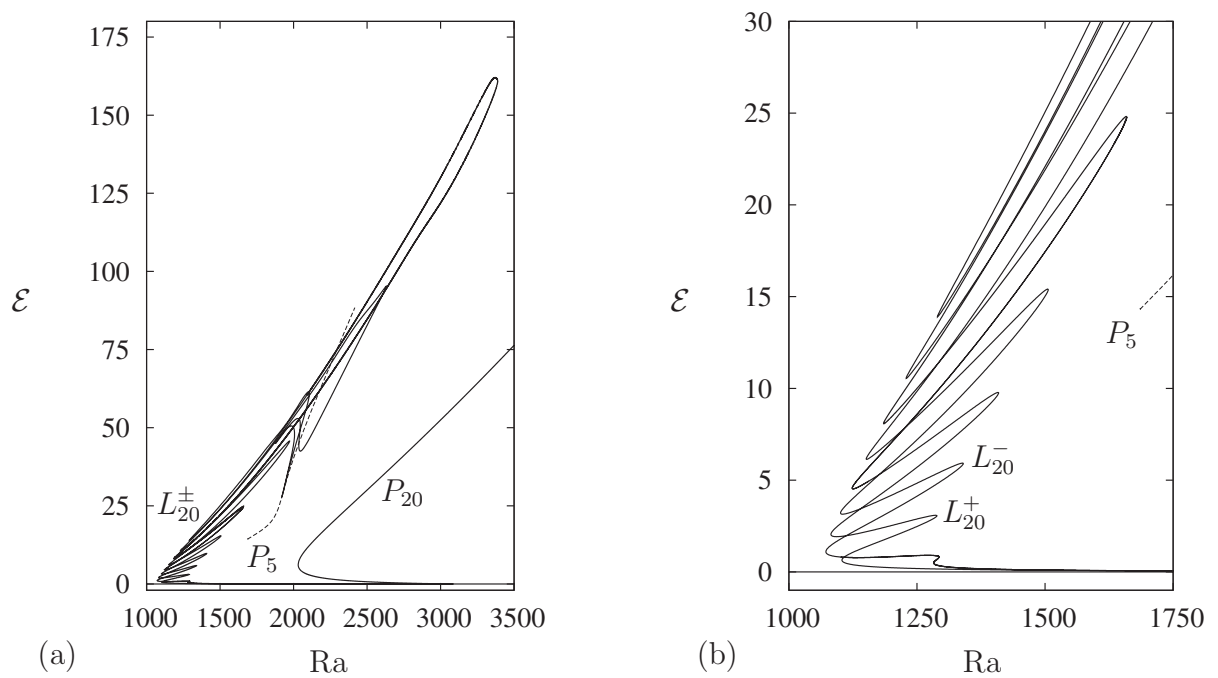

FIG. 1. (a) Bifurcation diagram showing the poloidal kinetic energy density $\mathcal{E}$ as a function of $\mathrm{Ra}$ for $\mathrm{Ta}=60, \sigma=0.1$, and $\Gamma \approx 26.6682$ with stress-free boundary conditions. The labels $L_{20}^{ \pm}$indicate the two convecton branches that emerge at finite amplitude from the primary branch $P_{20}$ of stationary periodic solutions with 20 wavelengths in the domain $\mathcal{D}$. These branches terminate together on $P_{5}$, a branch of periodic solutions with 5 wavelengths, shown dashed. (b) Zoom of the lower left part of (a) showing the first saddle-nodes on the snaking branches. For clarity, only a small part of the $P_{5}$ branch is included.

respect to a suitable origin) and the point symmetry $R_{c}$. The branch bifurcates subcritically and passes a saddle-node at $\mathrm{Ra} \approx 2032$ before extending monotonically toward large amplitude states. Two distinct branches of localized states, $L_{20}^{ \pm}$, bifurcate together at low amplitude from $P_{20}$ through a modulational instability. ${ }^{30,31}$ The branch $L_{20}^{+}$consists of $R_{x}$-symmetric solutions (therefore breaking $R_{c}$ ) while the opposite is the case for $L_{20}^{-}$. As one follows $L_{20}^{ \pm}$from their birth, the modulation becomes strongly nonlinear creating a pair of fronts connecting the convection state to the conduction state. These branches extend further into the subcritical regime than $P_{20}$ (in fact all $P_{n}$ ) indicating that their presence does not require bistability between the conduction state and a periodic state, and the most localized convectons are found near the very first saddle-node on each branch. Figures 2 and 3 show solutions at successive saddle-nodes along these branches through plots of the poloidal streamfunction $\psi$ and the zonal velocity $v$. The zonal velocity profile develops at the same time as the fronts. However, in contrast to the cellular structure within $\psi(x, z)$ the zonal velocity $v(x, z)$ develops on a large horizontal scale with practically no vertical structure. We see that the solution at the leftmost saddle-node consists of a small number of rolls ( 2 for $L_{20}^{+}$and 1 for $L_{20}^{-}$) surrounded by fluid with almost no poloidal flow embedded within a large scale negative zonal shear. This shear is generated by expulsion of zonal velocity gradients from regions of closed contours by the WeissRhines-Young mechanism, ${ }^{32,33}$ resulting in a staircase structure with overall negative shear gradient within the convecton, as required by the imposed periodic boundary conditions in the horizontal. ${ }^{18}$ As a result in the laboratory frame the fluid outside the convectons rotates faster than $\Omega$ but within the convectons it rotates more slowly. Evidently, the shear expulsion process enables the convectons to maintain their identity even in a sheared background. After the leftmost saddle-node both $L_{20}^{ \pm}$ undergo snaking as the solutions grow in length by nucleating new rolls near every left saddle-node. During this process, the convective structure remains trapped within a negative zonal shear zone and the snaking slants toward increasing Ra. The $L_{20}^{ \pm}$branches eventually terminate together on $P_{5}$, a branch of stationary periodic solutions with 5 pairs of counter-rotating rolls in $\mathcal{D}$. Only a portion of this branch is shown in Fig. 1 to avoid unnecessary clutter. The solution at the termination point on $P_{5}$, as well as the solution at the saddle-node of $P_{20}$ are shown in Fig. 4. At the termination point on $P_{5}$, the large scale structure in the zonal velocity $v$ disappears and the zonal shear is now confined to stagnation points between adjacent rolls.

The above results are closely related to the fact that the zonal velocity $v$ is a phase-like variable. As explained in Refs. 30 and 31 this fact has dramatic consequences for the dynamics of the system 


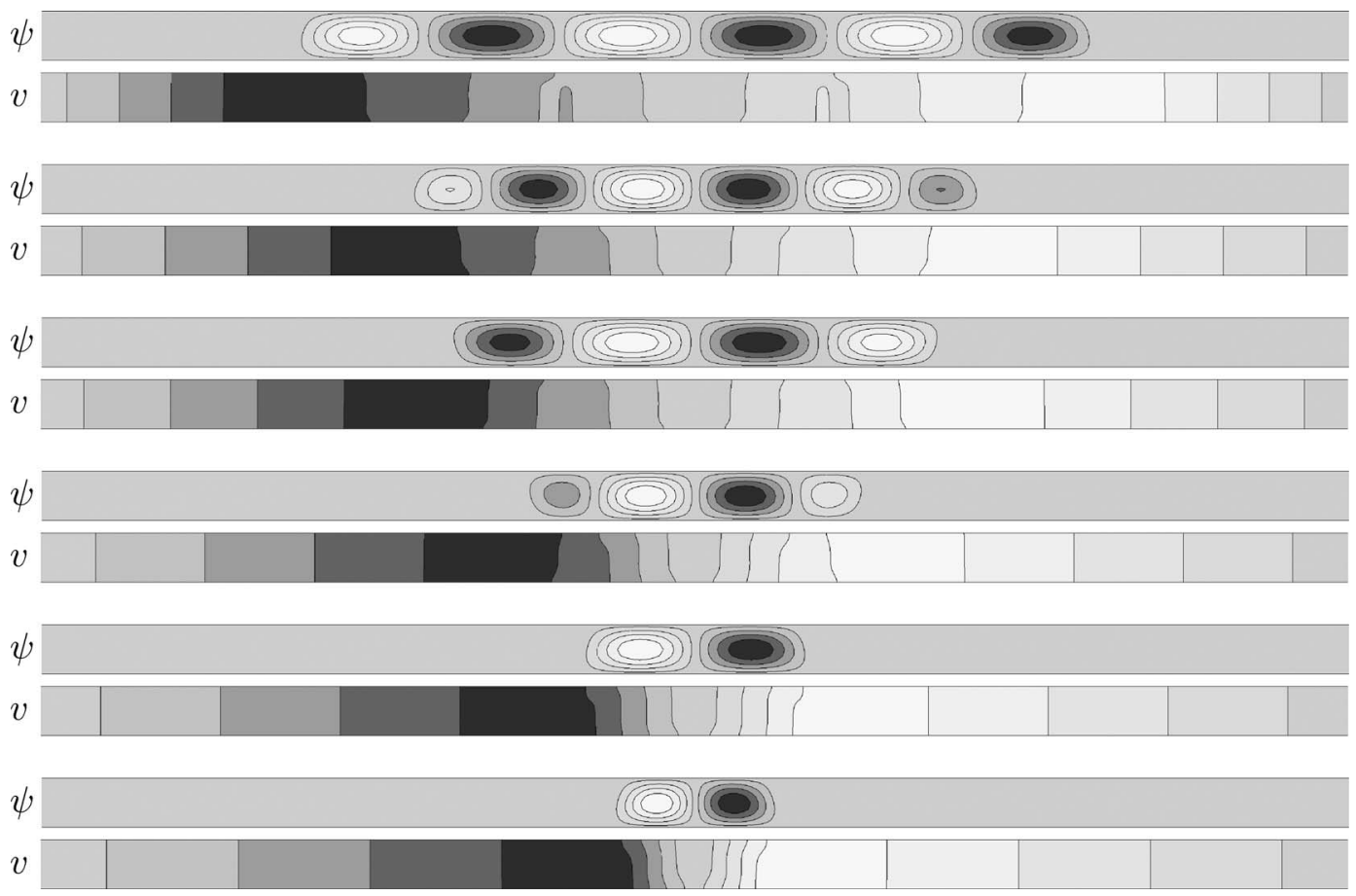

FIG. 2. Profiles of the $L_{20}^{+}$solutions taken at the first 6 saddle-nodes from the diagram in Fig. 1, starting from the lower left. The solutions are represented using contours of the poloidal streamfunction $\psi$ (upper panels) and the zonal velocity $v$ (lower panels). The contour values differ from one solution to the next although the contours are in all cases equidistributed between their maximum and minimum values, black denoting positive values (clockwise motion) and white negative values (counterclockwise motion). Likewise black (white) indicates regions of positive (negative) zonal velocity $v$.
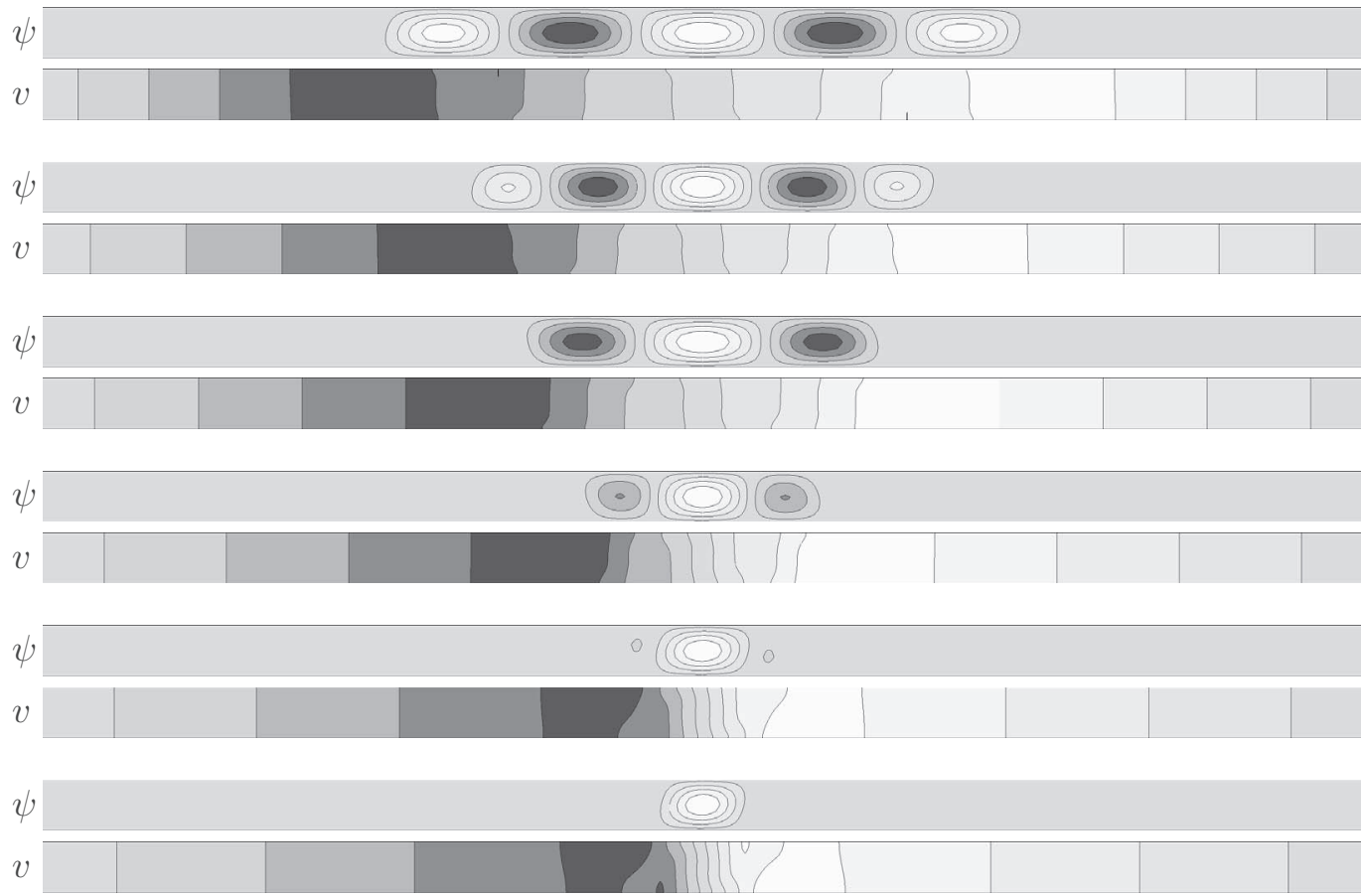

FIG. 3. As in Fig. 2 but for $L_{20}^{-}$. 


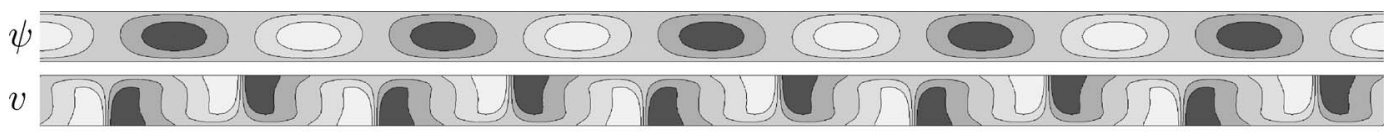

$\psi 0000000000000000000000000000000000000000$

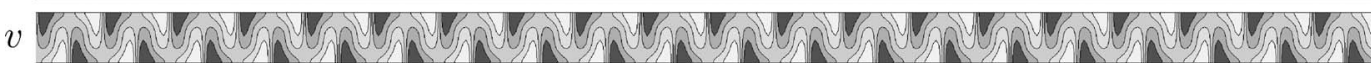

FIG. 4. Profiles of the $P_{20}$ solution close to its saddle-node (bottom panels) and of $P_{5}$ at the termination of the convecton branches (top panels). The solutions are represented using the same scheme as in Fig. 2. The evolution of these branches with $\mathrm{Ra}$ is shown in Fig. 1.

by forcing an interaction between small scales representing convection and large scales representing the zonal velocity. This is so even when scale separation is absent. ${ }^{34}$ The amplitude-dependent gradient expulsion resulting from the presence of closed contours changes the background state and requires a change in the Rayleigh number to maintain a localized state, thereby slanting the snaking structure in the bifurcation diagram. In other words, localized structures of smaller spatial extent exist at lower values of Ra than those of larger spatial extent. As a consequence, convectons exist over a larger range of parameter values and can be found even outside the bistability region between convection and conduction, i.e., the convectons are more subcritical than spatially periodic convection and hence are observable at parameter values at which periodic convection is absent. Indeed, convectons can be present even when periodic convection is supercritical. ${ }^{18}$

\section{NO-SLIP CONVECTONS}

As indicated in Sec. II, convectons are readily found in rotating convection with stress-free boundary conditions and occupy a substantial interval in parameter space, particularly for smaller Prandtl numbers. However, stress-free boundary conditions, while helpful for identifying the bifurcations responsible for the presence of convectons, are not realistic for laboratory experiments, and in this section we take a major step and report on the presence of similar states with no-slip boundary conditions at $z= \pm 1 / 2$,

$$
\psi=\psi_{z}=v=0
$$

These boundary conditions require zero velocity at the boundary, thereby changing the physical properties of the system, but preserve its equivariance with respect to the same symmetry group $O(2)$ $\times Z_{2}$. With the boundary conditions (11), Eq. (8) contains a source term,

$$
\frac{d}{d t} \bar{V}=\frac{\sigma}{\Gamma} \int_{-\Gamma / 2}^{\Gamma / 2}\left[\partial_{z} v\right]_{z=-1 / 2}^{1 / 2} d x
$$

where $\bar{V}$ still represents the average of $v$ over the domain. Thus $\bar{V}$ is no longer conserved, a consequence of wall friction. This friction vanishes in the stress-free case but not otherwise.

Figure 5 shows the bifurcation diagram for $\mathrm{Ta}=60, \sigma=0.1$, and periodic boundary conditions in the horizontal, obtained using homotopic continuation as described in Sec. IV. For these parameter values $k_{c} \approx 4.0575$, and hence $\Gamma \approx 30.9711$. Convection sets in through a stationary bifurcation at $\mathrm{Ra}_{c} \approx 3058$ producing a subcritical branch of stationary periodic solutions, $P_{20}$, with 20 pairs of counter-rotating rolls in the domain $\mathcal{D}$. Much as in the stress-free case, this branch passes a saddlenode at $\mathrm{Ra} \approx 2508$ before turning toward large amplitude states. A solution close to the saddle-node is shown in Fig. 6 (bottom panels). These solutions exhibit the same features as the periodic solutions with stress-free boundary conditions (Fig. 4), although the zonal shear is not advected near either wall where boundary layers are present that force $v$ to vanish. A secondary bifurcation occurs along $P_{20}$ at low amplitude and produces the branches $L_{20}^{ \pm}$of convectons, where the superscript + or refers to the same symmetry properties as for the stress-free convectons (Sec. II). These branches form through a similar mechanism to that for stress-free boundary conditions. Both are subcritical 

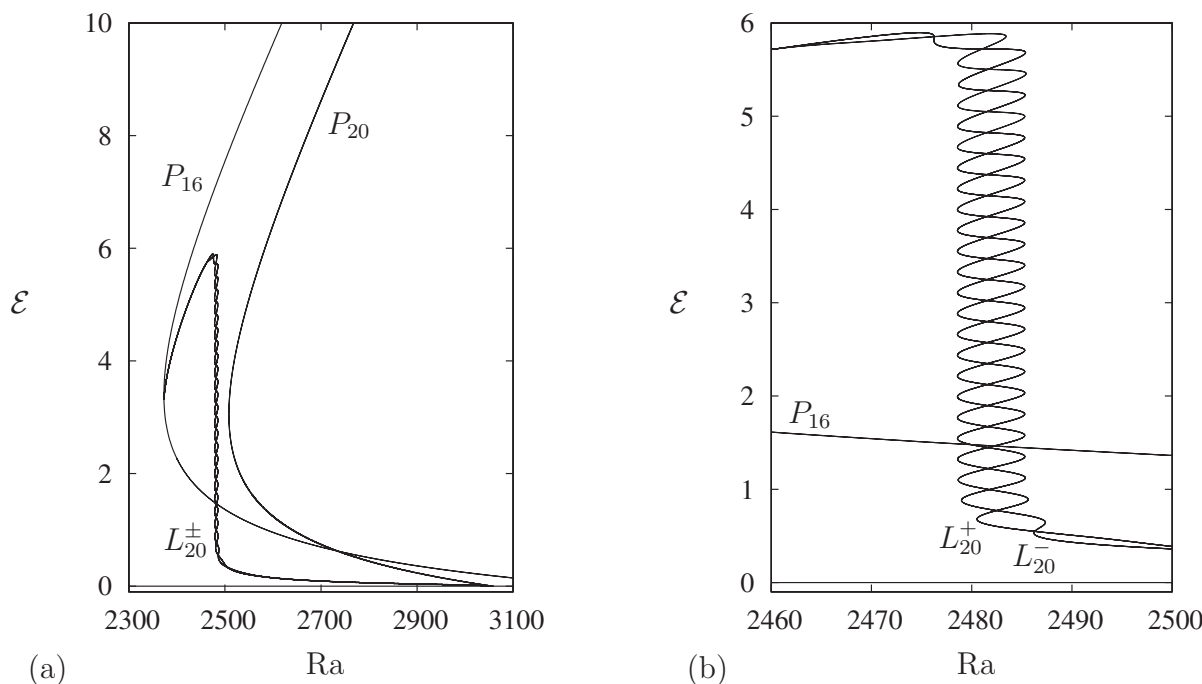

FIG. 5. (a) Bifurcation diagram showing the poloidal kinetic energy density $\mathcal{E}$ as a function of Ra for $\mathrm{Ta}=60, \sigma=0.1$, and $\Gamma \approx 30.9711$ with no-slip boundary conditions. The labels $L_{20}^{ \pm}$indicate the two convecton branches that emerge at finite amplitude from the primary branch $P_{20}$ of stationary periodic solutions with 20 wavelengths in the domain $\mathcal{D}$. These branches terminate together on $P_{16}$, a branch of periodic solutions with 16 wavelengths in $\mathcal{D}$. (b) Zoom of the snaking region in (a).

but this time they undergo standard snaking, without any slant, between $\mathrm{Ra} \approx 2478$ and $\mathrm{Ra} \approx 2486$ (Fig. 5). Representative solutions along the $L_{20}^{ \pm}$branches are shown in Figs. 7 and 8.

The solutions are most localized at the first saddle-node, where $L_{20}^{+}$consists in a 6-roll solution and $L_{20}^{-}$in a 7-roll solution. As one moves up either branch, the solutions grow spatially in the usual manner: after each back-and-forth excursion across the snaking region an extra pair of rolls is present, one roll on either side of the convective structure. The figures also reveal a major difference with the stress-free convectons already discussed: with no-slip boundary conditions the zonal velocity is strongly coupled to the poloidal flow and both fields vary on the same horizontal scale. Moreover, the convectons exhibit standard snaking in a well-defined Rayleigh number interval: both left and right saddle-nodes align for sufficiently broad structures. Once snaking is completed and the domain is filled the convecton branches $L_{20}^{ \pm}$exit the snaking region and reconnect with $P_{16}$ consisting of 16 pairs of rolls (Fig. 6). We expect that the snaking branches in Fig. 5 are connected by a series of crosslinks corresponding to drifting asymmetric solutions, much as in other problems of this kind. ${ }^{10}$ We expect such states to be unstable and have refrained from computing them.

It remains to offer an explanation for the presence of the 6- and 7-roll solutions at the bottom of the snaking region, instead of the more frequently seen 1- and 2-roll solutions. This type of non-minimal localized states has already been encountered in the context of the Swift-Hohenberg equation with competing quadratic and cubic nonlinearities by Burke ${ }^{35}$ who noted that the minimal

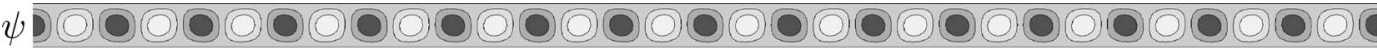

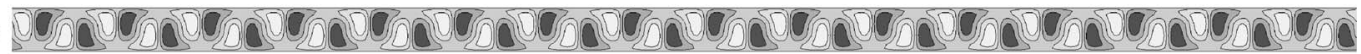

FIG. 6. Profiles of $P_{20}$ close to its saddle-node (bottom panels) and of $P_{16}$ at the termination of the convecton branches (top panels). The solutions are represented using contours of the poloidal streamfunction $\psi$ and the zonal velocity $v$. Contour values differ from one solution to the next but the contours are in all cases equidistributed between their maximum and minimum values, black denoting positive values (clockwise motion) and white negative values (counterclockwise motion). Likewise black (white) indicates regions of positive (negative) zonal velocity $v$. Parameters are those of Fig. 5. 


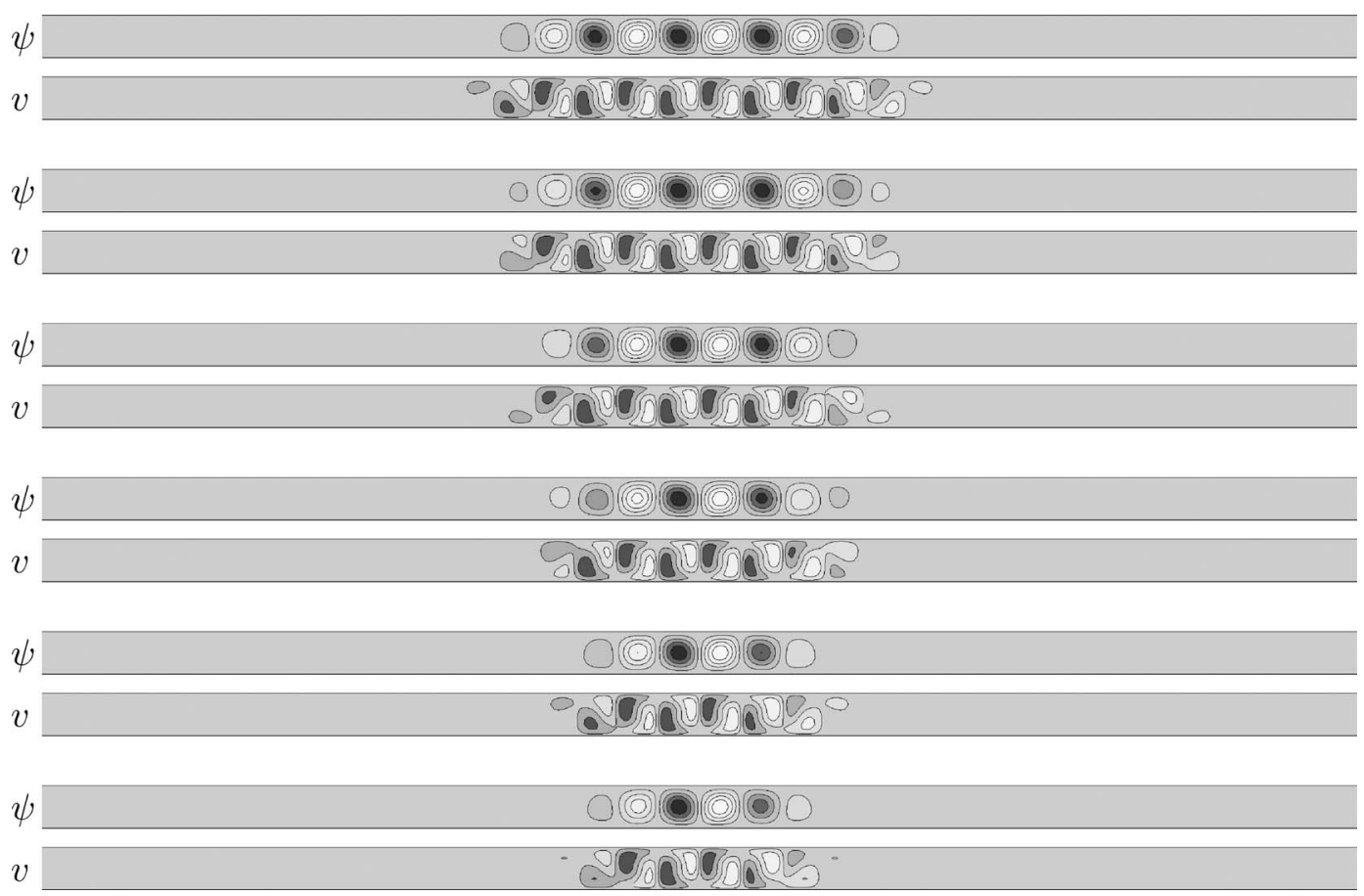

FIG. 7. Profiles of the six lowest saddle-node solutions on the $L_{20}^{+}$branch in Fig. 5, starting from the lower left, using the same representation as in Fig. 6.

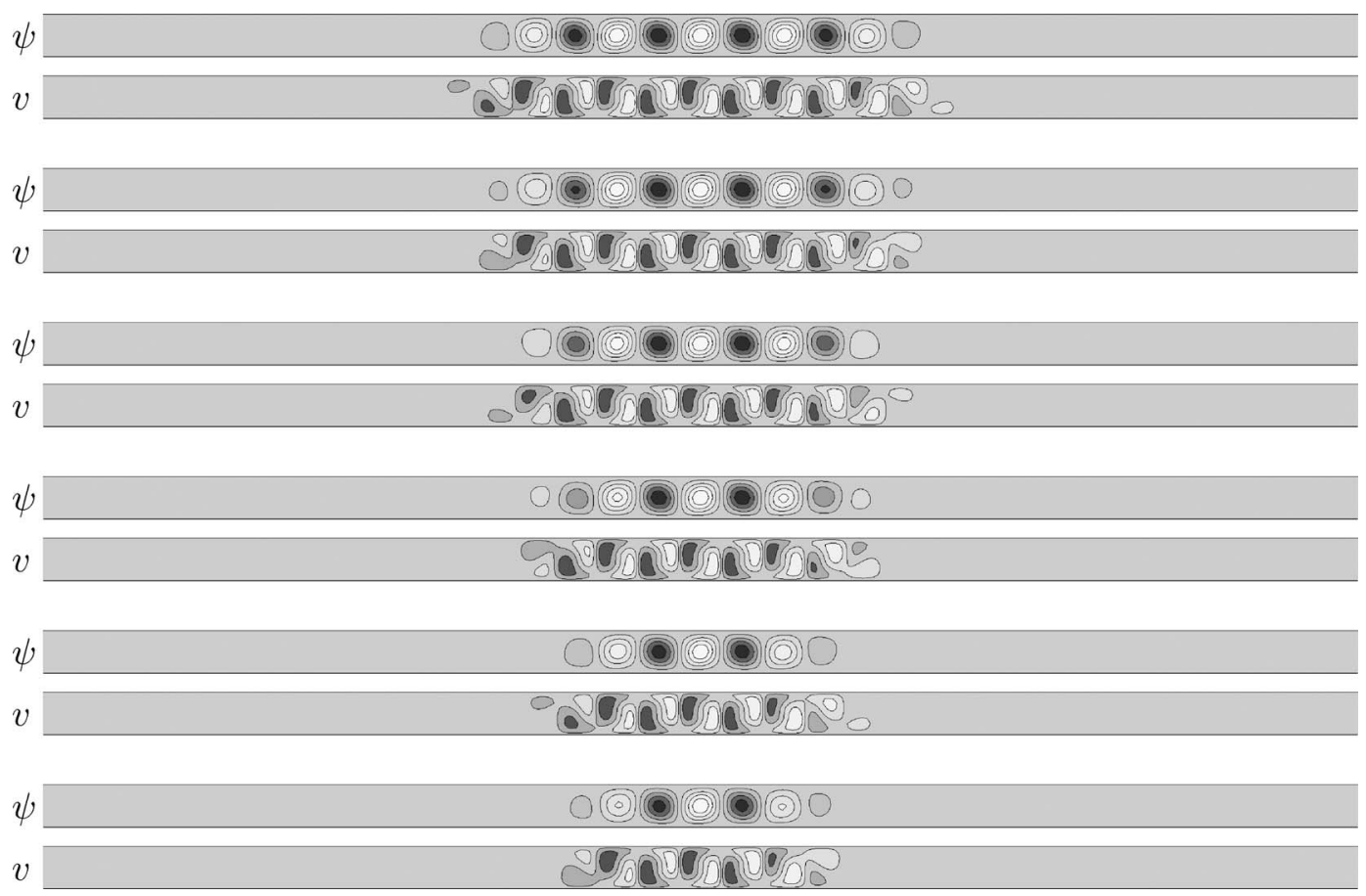

FIG. 8. As in Fig. 7 but for $L_{20}^{-}$. 
length of single pulse localized states in the snaking region depends on the coefficient $b_{2}$ of the quadratic nonlinearity. Burke ${ }^{35}$ found that as $b_{2}$ decreases the bottom saddle-nodes, associated with shorter localized solutions, are eliminated through a succession of hysteresis bifurcations. This process continues as $b_{2}$ decreases, gradually unzipping the snaking branches from the bottom until snaking is eliminated and the primary periodic branch becomes supercritical. We conjecture, therefore, that it is the weak subcriticality of the $P_{20}$ branch that is responsible in the present case for the absence of 1- and 2-roll localized states and that for smaller Prandtl numbers (stronger subcriticality) the snaking branches would add, via hysteresis bifurcations, additional saddle-nodes corresponding to shorter and shorter convectons. For fixed Ta, these saddle-nodes appear in cusps in the (Ra, $\sigma$ ) plane. Thus, it is the reduced subcriticality arising from the use of no-slip boundary conditions that is responsible for the elimination of the shortest convectons from the system.

We have also computed the leading eigenvalues along each of the odd and even convecton branches in Fig. 5 and found that convectons on the segments with positive slope are stable with respect to two-dimensional perturbations of like parity. This is not the case for stress-free boundary conditions since the primary steady state bifurcation is preceded, for these boundary conditions and parameter values, by several Hopf bifurcations from the conduction state. The resulting unstable eigenvalues are inherited by both periodic and localized convection, rendering all stationary solutions of either type unstable, at least at small amplitude. Thus, no-slip boundary conditions stabilize localized convection. These stability assignments follow those for the cubic-quintic Swift-Hohenberg equation, an equation that shares the symmetry $R_{z}$ with the present problem, ${ }^{12}$ and come about in exactly the same way.

\section{RELAXING THE CONSERVATION LAW}

To investigate the transformation from slanted to standard snaking presented in Secs. II and III, we compute solutions with boundary conditions that interpolate between stress-free and no-slip boundary conditions. Specifically, we impose

$$
\psi=(1-\beta) \psi_{z z} \pm \beta \psi_{z}=(1-\beta) v_{z} \pm \beta v=0
$$

at $z= \pm 1 / 2$, where $\beta \in[0,1]$ is a homotopy parameter. When $\beta=0$, we recover stress-free boundary conditions; when $\beta=1$ no-slip boundary conditions are applied. The signs in these conditions are determined by the requirement that friction is positive. Moreover, the conditions respect the symmetry properties of the system.

\section{A. Onset of convection}

To study the onset of convection with the mixed boundary conditions (13), we consider the linearized version of Eqs. (1)-(3),

$$
\begin{aligned}
\sigma^{-1} \nabla^{2} \psi_{t} & =\operatorname{Ra} \theta_{x}-\operatorname{Ta} v_{z}+\nabla^{4} \psi, \\
\theta_{t} & =\psi_{x}+\nabla^{2} \theta, \\
\sigma^{-1} v_{t} & =\text { Ta } \psi_{z}+\nabla^{2} v,
\end{aligned}
$$

and look for steady bifurcations yielding solutions with horizontal wavenumber $k$. The linearized equations can then be combined into a single equation for $\psi$,

$$
\left[\left(\partial_{z z}-k^{2}\right)^{3}+\operatorname{Ra} k^{2}+\operatorname{Ta}^{2} \partial_{z z}\right] \psi=0,
$$

with characteristic polynomial

$$
\left(\lambda^{2}+k^{2}\right)^{3}+\mathrm{Ta}^{2} \lambda^{2}=\operatorname{Ra} k^{2} .
$$

The three roots of polynomial (18) are $\lambda_{0}^{2}$, which is real positive, and a complex conjugate pair $\left(\lambda_{1}^{2}, \lambda_{1}^{* 2}\right)$. Steady convection sets in first for solutions consisting in only one roll in the vertical, 
allowing us to restrict attention to even solutions in $z$. The general form of these solutions is

$$
\begin{aligned}
& \psi=\cos \left(\lambda_{0} z\right) A+\left\{\cos \left(\lambda_{1} z\right) B+c . c .\right\}, \\
& \theta=i\left[\frac{k \cos \left(\lambda_{0} z\right)}{p_{0}} A+\left\{\frac{k \cos \left(\lambda_{1} z\right)}{p_{1}} B+c . c .\right\}+\frac{\mathrm{Ta}^{2} \cosh (k z)}{\mathrm{Ra}} C\right], \\
& v=-\operatorname{Ta}\left[\frac{\lambda_{0} \sin \left(\lambda_{0} z\right)}{p_{0}} A+\left\{\frac{\lambda_{1} \sin \left(\lambda_{1} z\right)}{p_{1}} B+c . c .\right\}+\sinh (k z) C\right],
\end{aligned}
$$

where $p_{n} \equiv k^{2}+\lambda_{n}^{2}$ and $A, B, C$ are constants to be determined. In order to satisfy the boundary conditions at $z= \pm 1 / 2$, the linear homogeneous system

$$
\mathbf{M}\left(A, B, B^{*}, C\right)^{T}=0
$$

must yield a nontrivial solution. In these expressions, we have set $\mathbf{M}=\left(\mathbf{M}_{0}, \mathbf{M}_{1}, \mathbf{M}_{1}^{*}, \mathbf{N}\right)$ with

$$
\begin{aligned}
& \mathbf{M}_{n}=\left(\begin{array}{c}
\cos \left(\lambda_{n} / 2\right) \\
(1-\beta) \lambda_{n}^{2} \cos \left(\lambda_{n} / 2\right)+\beta \lambda_{n} \sin \left(\lambda_{n} / 2\right) \\
{\left[(1-\beta) \lambda_{n}^{2} \cos \left(\lambda_{n} / 2\right)+\beta \lambda_{n} \sin \left(\lambda_{n} / 2\right)\right] / p_{n}} \\
\cos \left(\lambda_{n} / 2\right) / p_{n}
\end{array}\right), \quad n=0,1 \\
& 0 \\
& 0 \\
& \mathbf{N}=\left(\begin{array}{c}
(1-\beta) k \cosh (k / 2)+\beta \sinh (k / 2) \\
\frac{\mathrm{Ta}^{2}}{\operatorname{Ra} k} \cosh (k / 2)
\end{array}\right)
\end{aligned}
$$

The condition that the determinant of $\mathbf{M}$ vanishes yields the critical Rayleigh number $\mathrm{Ra}_{c}$ and the corresponding critical wavenumber $k_{c}$ as functions of Ta and $\beta$.

Figure 9 shows the dependence of $\mathrm{Ra}_{c}$ and $k_{c}$ on $\beta$ when $\mathrm{Ta}=0,20,60$, and 100. The critical Rayleigh number $\mathrm{Ra}_{c}$ increases with $\beta$ for small Taylor numbers implying that at small rotation rates convection is easier to trigger with stress-free boundary conditions. However, for sufficiently large Taylor numbers (typically of the order of 100 ), $\mathrm{Ra}_{c}$ first decreases as $\beta$ increases, reaching a minimum close to $\beta=1$ before increasing abruptly even closer to $\beta=1$. This result indicates that for strong enough rotation rates, wall friction is actually destabilizing. This reversal in tendency between the small and large rotation rates also impacts the critical wavelength of the unstable pattern: for slow rotation, as wall friction increases, the wavenumber $k_{c}$ of the convective structure increases. For large rotation rates, however, increasing $\beta$ leads to smaller wavenumbers of the unstable mode. Overall, increasing rotation favors vertical motion in the fluid rather than horizontal motion as a consequence of the Taylor-Proudman theorem and leads to the emergence of narrower structures, and hence of

(a)

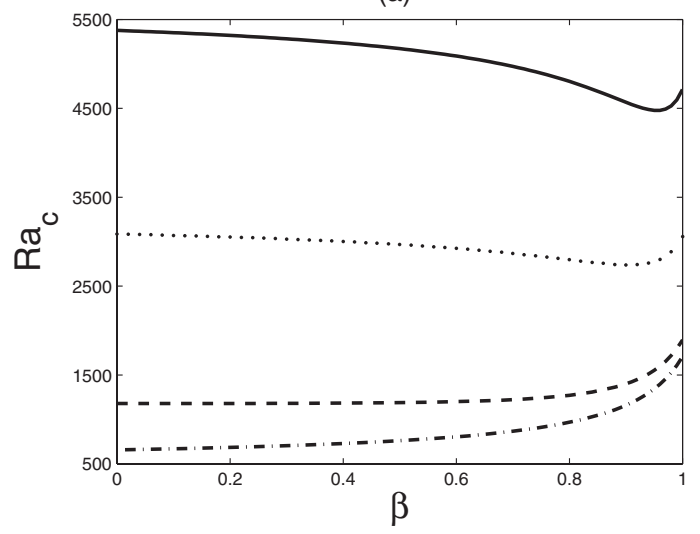

(b)

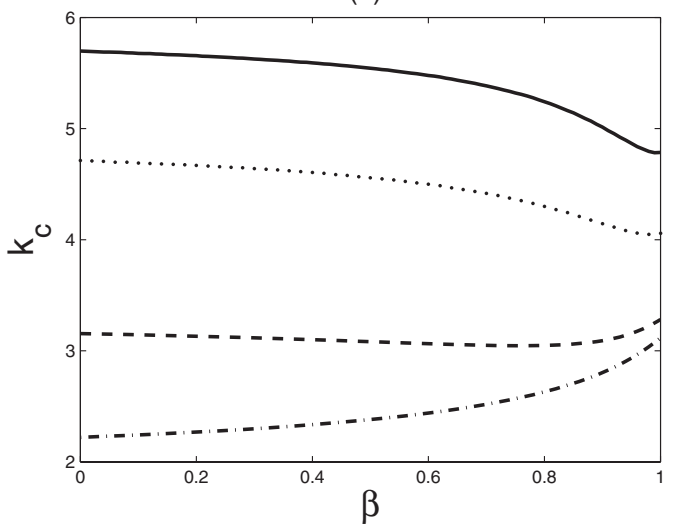

FIG. 9. (a) $\mathrm{Ra}_{c}$ and (b) $k_{c}$ as functions of $\beta$. Solid line: $\mathrm{Ta}=100$. Dotted line: $\mathrm{Ta}=60$. Dashed line: $\mathrm{Ta}=20$. Dashed-dotted line: $\mathrm{Ta}=0$. 
larger wavenumbers. However, no-slip boundary conditions $(\beta=1)$ exert a stronger constraint on the flow than stress-free ones $(\beta=0)$, so the effect described by the Taylor-Proudman theorem is less effective when no-slip boundary conditions are applied. These arguments explain why the curves in Fig. 9(b) approach one another as $\beta$ increases, leading to lower critical wavenumbers for large rotation rates.

\section{B. Damping of the large scale mode}

As one moves away from stress-free boundary conditions, i.e., $\beta$ becomes nonzero, the conservation law is broken. For $\beta=0$, the large scale mode is given by $(\psi, \theta, v)=(0,0,1)$. This mode is marginal when $\beta=0$ but this is no longer so when $\beta$ departs from zero and the large scale mode becomes damped. According to Eqs. (1)-(3) the large scale mode satisfies

$$
\begin{aligned}
- \text { Ta } v_{z}+\partial_{z z}\left(\partial_{z z}-\sigma^{-1} \mu\right) \psi & =0, \\
\text { Ta } \psi_{z}+\left(\partial_{z z}-\sigma^{-1} \mu\right) v & =0,
\end{aligned}
$$

where $\mu$ is the growth rate of the mode. Combining Eqs. (23) and (24) together we have

$$
\mathrm{Ta}^{2} v_{z z}=-\left(\partial_{z z}-\sigma^{-1} \mu\right)^{2} v_{z z} .
$$

There are in total six linearly independent solutions for Eq. (25) but only the modes $v$ that are even in $z$ are relevant. The general form of the solution is thus

$$
\begin{aligned}
& \psi=\frac{\sigma^{-1} \mu}{\mathrm{Ta}} A z+\left\{i \lambda_{1}^{-1} B \sin \left(\lambda_{1} z\right)+c . c .\right\}, \\
& v=A+\left\{B \cos \left(\lambda_{1} z\right)+\text { c.c. }\right\},
\end{aligned}
$$

where $\lambda_{1}^{2}=-\sigma^{-1} \mu+i$ Ta. The boundary conditions at $z= \pm 1 / 2$ imply that

$$
\begin{aligned}
\frac{\sigma^{-1} \mu}{2 \mathrm{Ta}} A+\left\{\frac{i \sin \left(\lambda_{1} / 2\right)}{\lambda_{1}} B+c . c .\right\} & =0, \\
\frac{\beta \sigma^{-1} \mu}{\mathrm{Ta}} A+\left\{i\left[(\beta-1) \lambda_{1} \sin \left(\frac{\lambda_{1}}{2}\right)+\beta \cos \left(\frac{\lambda_{1}}{2}\right)\right] B+c . c .\right\} & =0, \\
\beta A+\left\{\left[(\beta-1) \lambda_{1} \sin \left(\frac{\lambda_{1}}{2}\right)+\beta \cos \left(\frac{\lambda_{1}}{2}\right)\right] B+c . c .\right\} & =0 .
\end{aligned}
$$

The dependence of $\sigma^{-1} \mu$ on the parameters Ta and $\beta$ can be obtained by applying the solvability condition to this linear homogeneous system. The results are plotted in Fig. 10. The growth rate is zero for $\beta=0$ indicating the presence of a large scale, marginally stable zonal velocity mode for stress-free boundary conditions. The growth rate then decreases monotonically as $\beta$ increases. In addition, for a fixed $\sigma, \mu$ decreases as the Taylor number Ta increases as shown in Fig. 10 but the numerical values and the slopes of $\sigma^{-1} \mu$ (with respect to $\beta$ ) are close to each other when $\beta$ is small. Indeed, for small $\beta$, the dependence of $\sigma^{-1} \mu$ on $\beta$ takes the form

$$
\sigma^{-1} \mu=-2 \beta+O\left(\beta^{2}\right) .
$$

This relation has consequences for the weakly nonlinear regime, as discussed next.

\section{Weakly nonlinear theory}

The above results suggest that when the magnitude of $\beta$ is of $O\left(\epsilon^{2}\right), 0<\epsilon \ll 1$, and $\epsilon^{2}$ measures the difference between $\mathrm{Ra}$ and $\mathrm{Ra}_{c}$, a linear damping term should be added into the equation derived 


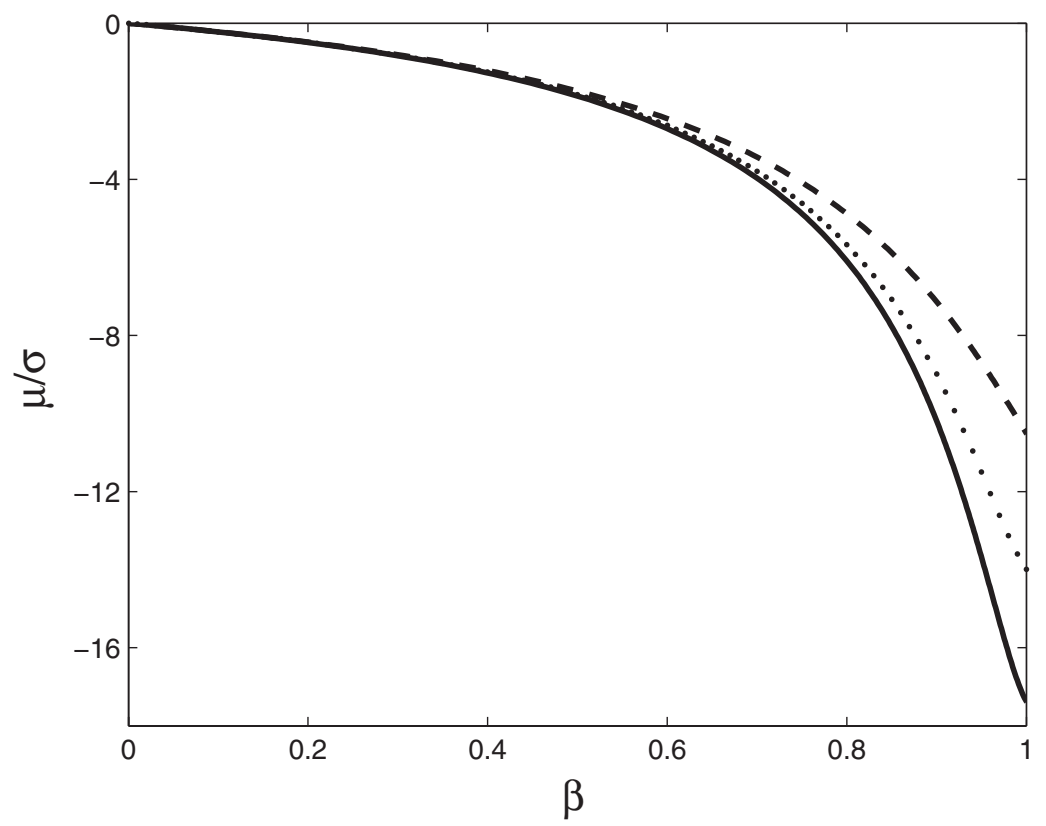

FIG. 10. Dependence of $\sigma^{-1} \mu$ on $\beta$. Solid line: Ta $=100$. Dotted line: $\mathrm{Ta}=60$. Dashed line: $\mathrm{Ta}=20$.

for the large-scale mode by Cox and Matthews. ${ }^{31}$ With $\beta=\epsilon^{2} \beta_{2}$ and the scaling used in Refs. 18 and 31 , the resulting modulation equations for rotating convection take the form

$$
\begin{aligned}
\eta A_{T} & =r A+A_{X X}-\frac{\left(1-\xi^{2}\right)}{2}|A|^{2} A-\xi A V_{X}, \\
V_{T} & =-\lambda V+V_{X X}+\xi\left(|A|^{2}\right)_{X}
\end{aligned}
$$

where $\lambda \equiv 24 p \beta_{2}$ is the scaled linear decay rate of the large scale mode, and $p \equiv k^{2}+\pi^{2}$.

In the stationary case, the gradient of the large scale mode can be expressed as

$$
V_{X}=\xi\left(\frac{1}{2} \int_{\mathbb{R}} e^{-|Y|}\left|A\left(X+\lambda^{-1 / 2} Y\right)\right|^{2} d Y-|A|^{2}\right)
$$

and the stationary equation for $A$ takes the nonlocal form

$$
A_{X X}+\left(r-\frac{\xi^{2}}{2} \int_{\mathbb{R}} e^{-|Y|}\left|A\left(X+\lambda^{-1 / 2} Y\right)\right|^{2} d Y\right) A-\frac{1-3 \xi^{2}}{2}|A|^{2} A=0
$$

Here the domain of integration has been extended to the whole real line on the assumption that the damping length scale $\lambda^{-1 / 2}$ is in fact much larger than all the other length scales in the problem. The new length scale renders the problem nonlocal and interpolates between local and globally coupled formulations of the weakly nonlinear problem. When $\lambda$ is large or equivalently $\beta$ is an order of magnitude larger than $O\left(\epsilon^{2}\right)$, the large scale mode $V$ becomes highly damped and the governing modulation equation reduces to the cubic real Ginzburg-Landau equation. Figure 11 shows the bifurcation diagrams computed from Eqs. (32) and (33) for several different sets of values of the parameters $\sigma$ and Ta and several different values of $\beta$. The branches are calculated using AUTO $^{36}$ 

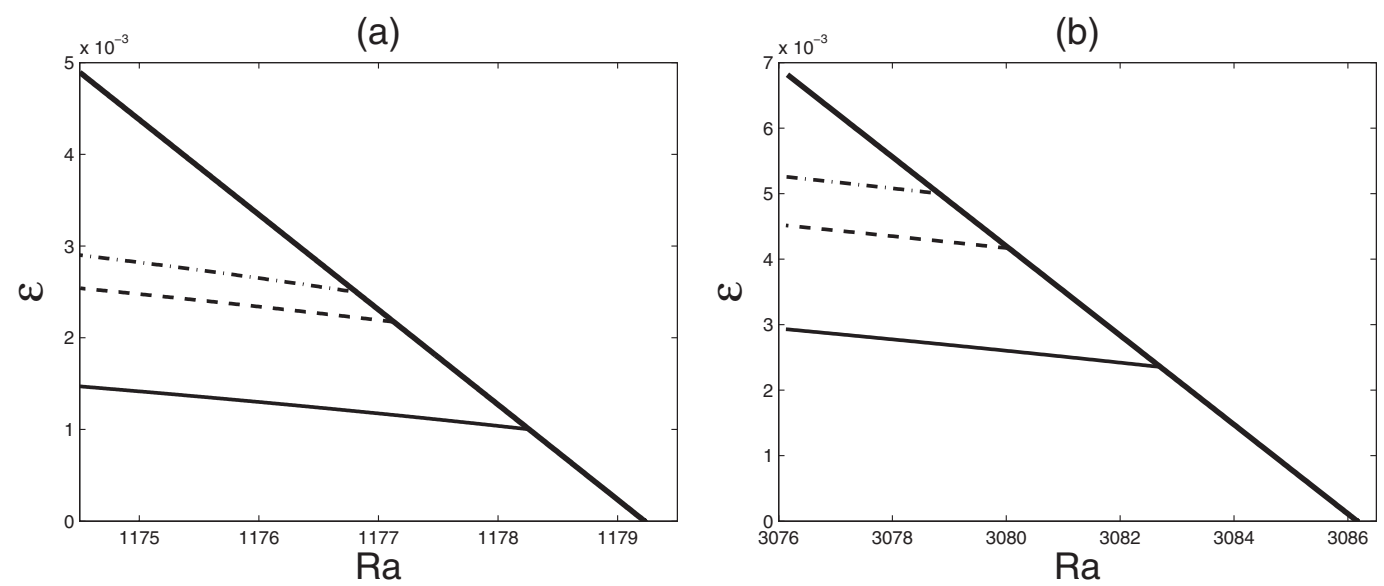

(c)

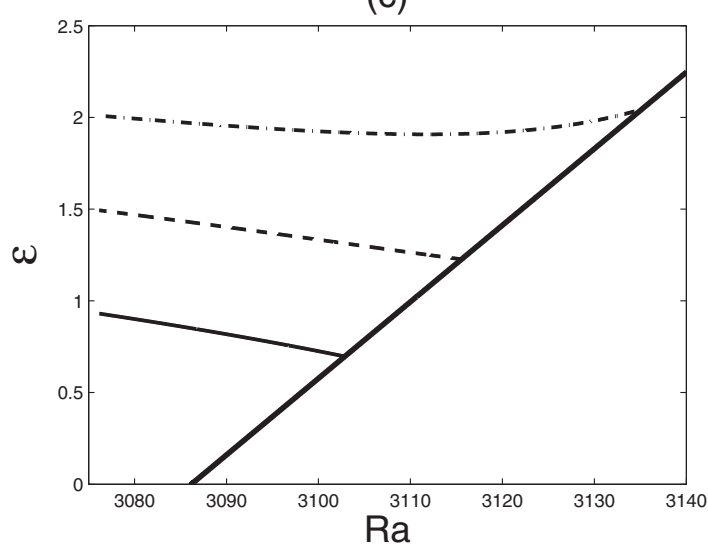

FIG. 11. Bifurcation diagrams showing poloidal kinetic energy density $\mathcal{E}$ as a function of Ra computed from the modulation equations (32) and (33) with $\Gamma=20 \lambda_{c}$ for (a) $\mathrm{Ta}=20, \sigma=0.1$, (b) $\mathrm{Ta}=60, \sigma=0.1$, and (c) $\mathrm{Ta}=60, \sigma=0.6$. Thick solid line: periodic state. Thin lines: spatially modulated states (solid line: $\beta=0$, dashed line: $\beta=0.05$, dashed-dotted line: $\beta=0.1$.

with the vertical axis representing the poloidal kinetic energy density

$$
\mathcal{E}=\frac{\epsilon^{2}\left\langle|A|^{2}\right\rangle}{6 k^{2}}+\frac{\epsilon^{3}\left\langle\operatorname{Im}\left[A^{*} A_{X}\right]\right\rangle}{6 \sqrt{3} p^{3 / 2} k}+\frac{\epsilon^{4}\left\langle\left|A_{X}\right|^{2}\right\rangle}{72 p^{2} k^{2}}+O\left(\epsilon^{5}\right)
$$

truncated at fourth order in $\epsilon$. Here $\langle\cdots\rangle \equiv(\epsilon \Gamma)^{-1} \int_{-\epsilon \Gamma / 2}^{\epsilon \Gamma / 2}(\cdots) d X$. The value of the horizontal coordinate, the Rayleigh number, is obtained via $\mathrm{Ra}=\mathrm{Ra}_{c}+\epsilon^{2} r$. In order to compare the results for different $\beta$, we shift the branches with nonzero $\beta$ horizontally in such a way that the periodic branches (thick black lines) all align with the $\beta=0$ periodic branch. The periodic branches (thick solid lines) all have wavenumber $k=k_{c}$. The thin lines correspond to the first spatially modulated solutions that bifurcate from the periodic solution, each for a different value of $\beta$ : solid lines correspond to $\beta=0$, dashed lines correspond to $\beta=0.05$, and dashed-dotted lines correspond to $\beta=0.1$. In all cases, the secondary bifurcation points move to higher energy as $\beta$ increases and the bifurcation behavior reduces to that predicted by the cubic real Ginzburg-Landau equation when $\beta$ is large: the first secondary bifurcation occurs at $r=-\frac{2 \pi^{2}}{\epsilon^{2} \Gamma^{2}}$ in the subcritical case and no secondary bifurcation is present in the supercritical case. For example, in Fig. 11(c) the primary bifurcation is supercritical and as $\beta$ increases the interval of stable periodic states grows and the destabilization due to the large scale mode moves to large amplitudes where weakly nonlinear theory is no longer valid. Results of the type presented in Fig. 11 provide predictions for the location of secondary bifurcations to localized states and yield good agreement with the full numerical computations that follow. 

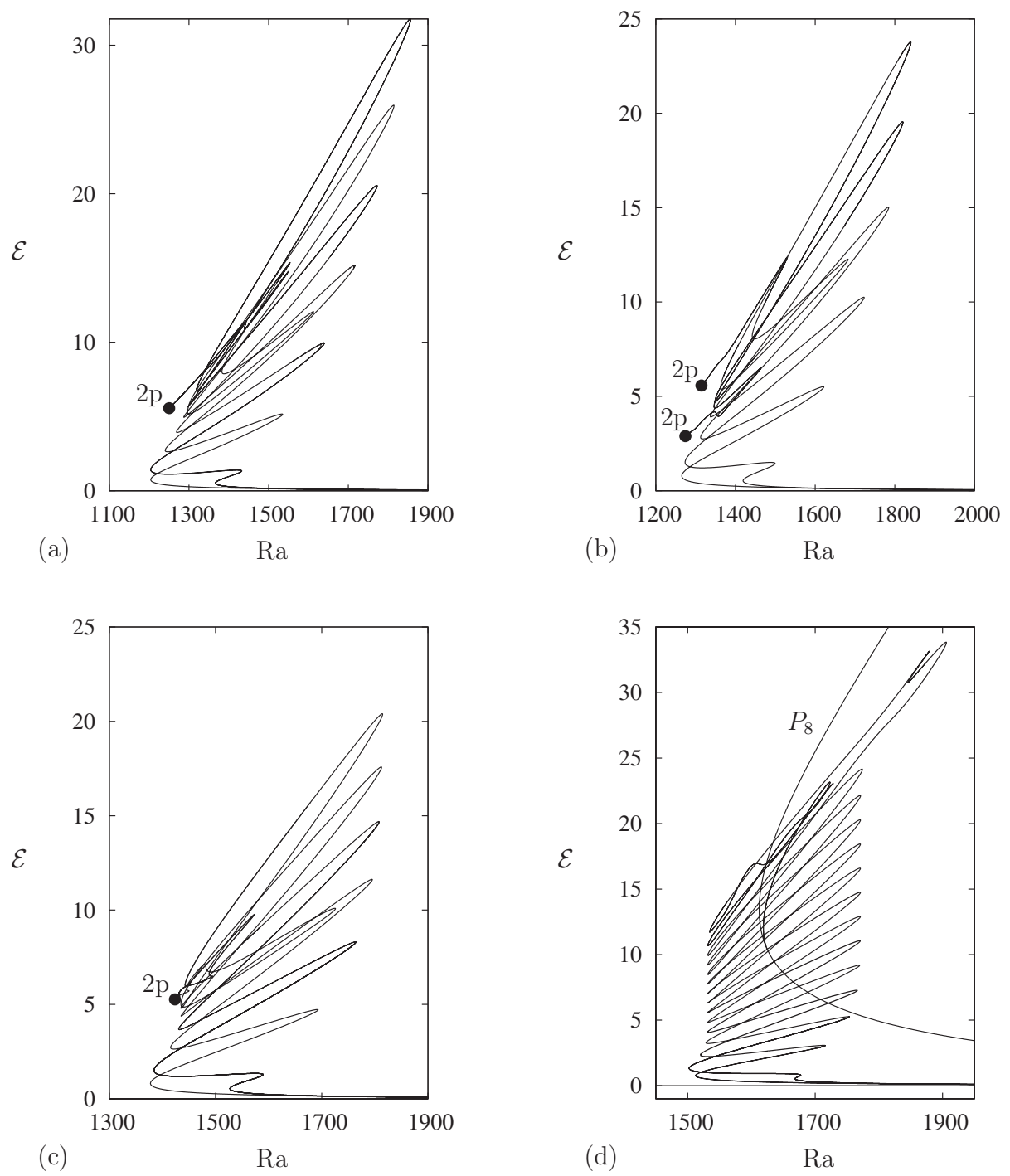

FIG. 12. Bifurcation diagrams showing the poloidal kinetic energy density $\mathcal{E}$ for odd and even convectons for $\mathrm{Ta}=60$, $\sigma=0.1$ and (a) $\beta=0.1$, (b) $\beta=0.2$, (c) $\beta=0.4$, and (d) $\beta=0.6$, all computed on 20 wavelength periodic domains. The solid dot, labeled $2 \mathrm{p}$, indicates the termination of the localized states on a branch of 2-pulse states. For $\beta=0.6$, the convecton branches terminate on $P_{8}$ instead.

\section{Nonlinear results}

Fully nonlinear results for $\beta=0.1,0.2,0.4$, and 0.6 at $\mathrm{Ta}=60$ and $\sigma=0.1$, obtained using homotopic continuation from the stress-free results, are shown in Fig. 12. For $\beta=0.6$, homoclinic snaking is found resembling that obtained for no-slip boundary conditions $(\beta=1)$, i.e., standard snaking between two well-defined values of Ra despite some initial misalignment. However, as $\beta$ decreases a transition takes place: for $\beta=0.1,0.2$, and 0.4 snaking is no longer complete and the convecton branches terminate on a branch of equidistant 2-pulse states at points labeled $2 p$ in Fig. 12. The 2-pulse state develops from the 1-pulse state gradually, as in other problems of this type, ${ }^{18,37}$ once the 1-pulse state has reached maximum amplitude, a process that is reflected in the shorter but tighter excursions of the branch toward the end of the 1-pulse branch (Fig. 12). During this process the central roll (or rolls) weakens and starts to fade away, gradually breaking the convecton into two nearby pieces. As one follows the 1-pulse branch, these two pieces remain nearby but their 

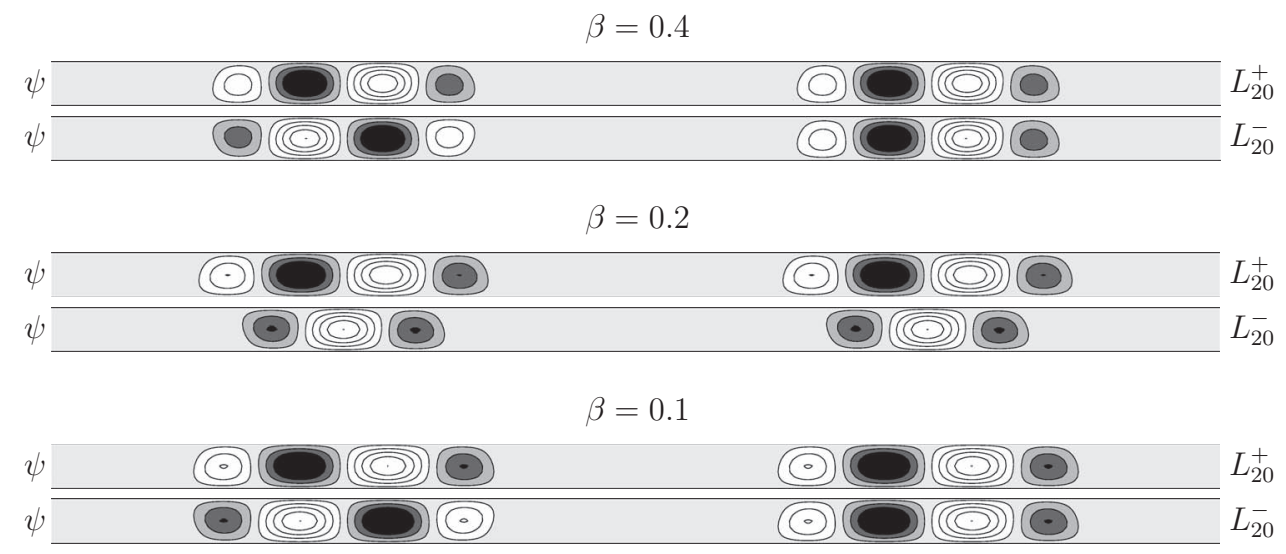

FIG. 13. Solutions in a 20 wavelength domain at the termination points $2 \mathrm{p}$ of the branches of localized states when $\mathrm{Ta}=60$, $\sigma=0.1$, and $\beta=0.1,0.2$, and 0.4 , indicated by solid dots in Fig. 12.

separation increases, slowly at first and then much more rapidly just before the termination of the branch, reaching $\Gamma / 2$ at the termination point $2 p$. Figure 13 shows the resulting equidistant 2-pulse states at $2 p$ for the three examples in Fig. 12. The resulting branches of equidistant 2-pulse states are omitted from Fig. 12. These branches are expected to bifurcate from the first branch of periodic states at a larger amplitude than the 1-pulse solutions and to snake in the same region as the 1-pulse states. ${ }^{28,38}$ However, the connection between the 1- and 2-pulse branches observed here has not been observed in other systems. We surmise that it forms as a result of a tangency between these branches that forms as $\beta$ decreases. This tangency breaks the 1-pulse branch into two disconnected pieces, each of which terminates on the 2-pulse branch; only one of these, the one that snakes, is shown in the figure. Because the equidistant 2-pulse states bifurcate from the periodic states after the 1-pulse states they are expected to be unstable and we have not found any stable states of this type.

In order to understand the origin of the splitting of the convectons that takes place as $\beta$ decreases, we examine the role played by wall stress. When $\beta=0$ the zonal velocity $v$ associated with a convecton is dominated by large scale variation on the scale of the domain that peaks (dips) at the left (right) of the convecton implying an overall negative shear across the convecton. ${ }^{18}$ As $\beta$ departs from $0, v$ ceases to be phase-like but the symmetries of the convecton (either $R_{c}$ or $R_{x}$ ) imply that $v$ must have zero mean. In addition, as friction becomes significant, vertical gradients of zonal velocity proportional to $\beta v /(1-\beta)$ develop. These gradients in turn shear the rolls in the $y$ direction through the action of the Coriolis term in Eq. (1). As a result, as the localized structure grows in space, its top and bottom boundaries are subject to stronger and stronger forcing from the zonal boundary layer while its core continues to rotate substantially more slowly than the boundaries. This forcing tends to spin up the convecton implying a reduction in zonal shear across the structure in comparison to the stress-free case. At a critical Rayleigh number, the boundary layer forcing becomes too strong and the convecton cannot maintain integrity and splits into two shorter convectons. This transition is only observable at intermediate values of $\beta$. Indeed, when $\beta$ is too large, no large scale variations of $v$ are created, while for $\beta$ too small, wall friction is not strong enough to generate sufficient stress on the convecton to break it apart.

When the conservation law is broken by the boundary conditions, another important change in the slanted snaking that prevails when $\beta=0$ is observed. Figure 12 shows that when $\beta$ is still relatively small the snaking is neither slanted nor standard. Instead, the convecton branches start out as if slanted snaking were to take place but with increasing amplitude and hence Rayleigh number the snaking transitions from slanted snaking to something like standard snaking. In the following, we refer to this transition as curved snaking. This transition appears at low values of $\beta$ but competes here with the pulse-breaking mechanism described above. To study the transition in 


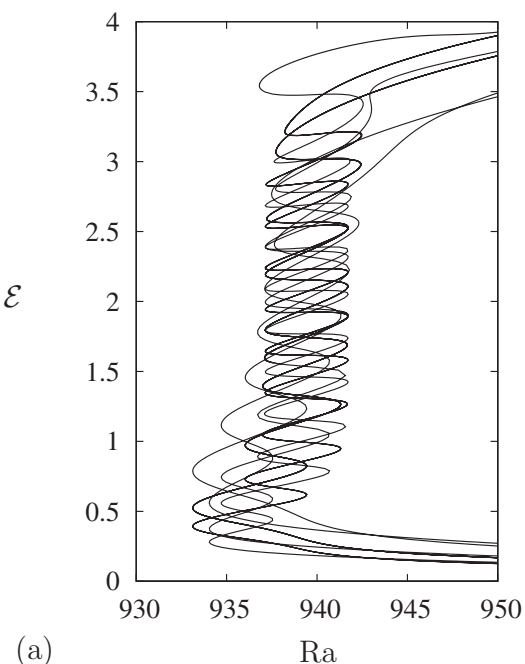

(a)

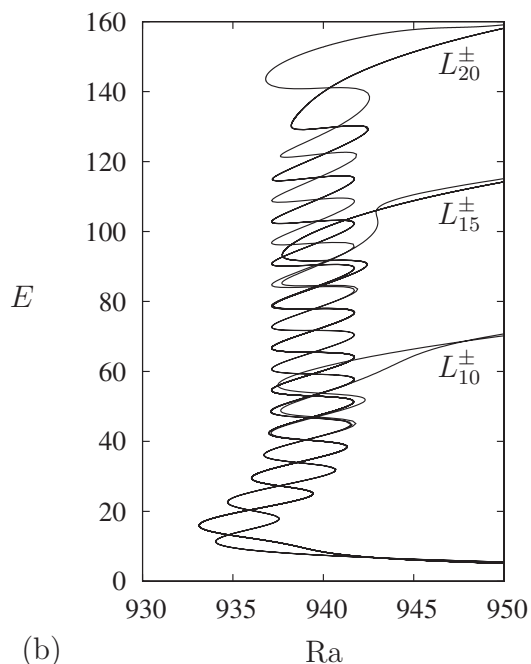

(b)

$\mathrm{Ra}$

FIG. 14. Bifurcation diagram for the branches of localized states for Ta $=20, \sigma=0.1$, and $\beta=0.4$ in terms of (a) the poloidal kinetic energy density $\mathcal{E}$ and (b) the total poloidal kinetic energy $E=\Gamma \mathcal{E}$. The labels $L_{10}^{ \pm}, L_{15}^{ \pm}$, and $L_{20}^{ \pm}$refer, respectively, to localized states in 10,15 , and 20 wavelength domains. The domains are meshed in the same way in all cases, using 20,30, and 40 elements for $\Gamma=10 \lambda_{c}, 15 \lambda_{c}$, and $20 \lambda_{c}$, respectively, each element having $15 \times 13$ points.

more detail, we therefore suppress this competition by decreasing the rotation rate so that the zonal velocity provides a weaker feedback to the rolls and does not lead to the splitting of the convecton. Figure 14 shows the bifurcation diagram obtained for $\mathrm{Ta}=20, \sigma=0.1, \beta=0.4$ and different domain sizes using two different representations: the poloidal kinetic energy density $\mathcal{E}$ and the total poloidal kinetic energy $E=\Gamma \mathcal{E}$. For these values of the Prandtl and Taylor numbers, none of our simulations show transitions to 2-pulse states and the localized states are broader than at higher Taylor numbers, a reflection of the increase in critical wavelength at relatively small rotation rates. ${ }^{18}$ Figure 14 captures well the phenomenon of curved snaking and compares it for different domain sizes. In the presence of stress-free boundary conditions, the system admits a conservation law and it proved useful to compare solutions using averaged quantities such as $\mathcal{E}$. This approach proved effective at capturing the slope of the slanted branches and helped establish the conclusion that in the presence of a conserved quantity the convecting region at fixed Rayleigh number always fills the same fraction of the available domain, regardless of the domain size, provided only that it is finite. ${ }^{17,18}$ For example, at a specific value of the Rayleigh number, 3-roll localized solutions may form in one domain, while 6-roll convectons would form in a domain twice as large (see Figs. 4 and 5 in Ref. 18). This statement no longer holds when the conservation law is broken (Fig. 14(a)). Instead, total quantities, such as $E$, are better indicators for comparing solutions (Fig. 14(b)). In the latter case, we observe that the snaking branches match virtually perfectly, regardless of domain size, at least until the domain is almost filled. This observation makes it clear that without the conservation law, it is the length of the convecton that is independent of the domain size, provided only that the domain is sufficiently large. Consequently, curved snaking is not a domain size effect and the dichotomy between the behavior of systems with and without a conserved quantity is real and fundamental. For any domain size, the convecton branches initially slant, but the slant decreases with increasing Rayleigh number and the snaking structure approaches standard snaking as the domain fills up. As a result, if the domain size is too small, the snaking will appear curved everywhere whereas if it is sufficiently long, the upper part will not be curved and will resemble standard snaking. This curvature is less pronounced when $\beta$ is small but becomes more abrupt as $\beta$ increases, leading to a faster transition to regular snaking as exemplified for $\mathrm{Ta}=20$ by Fig. 15 . For this Taylor number, the snaking region shrinks with increasing $\beta$ and ultimately disappears before the no-slip case $\beta=1$ is reached. 


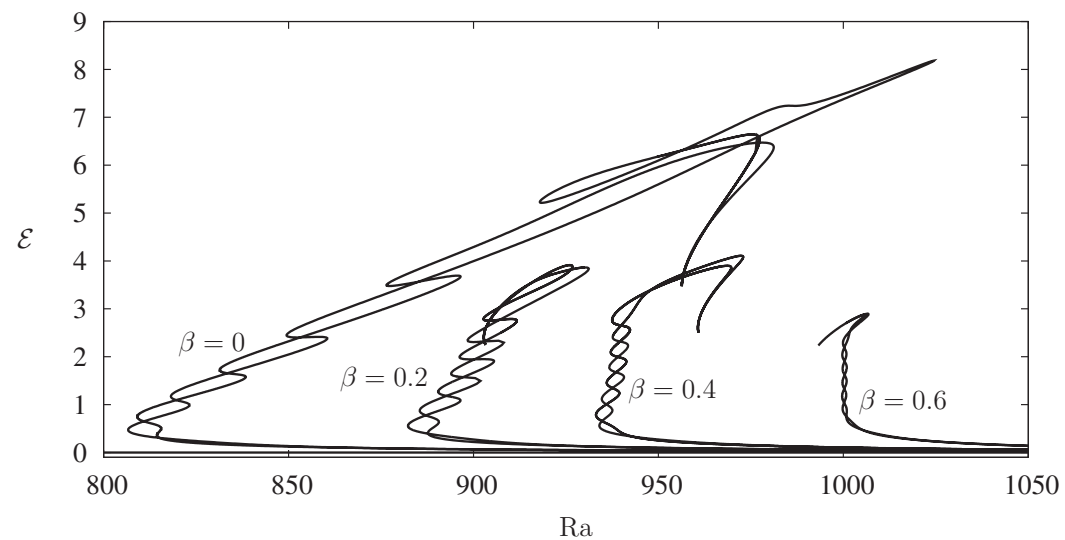

FIG. 15. Bifurcation diagrams representing the poloidal kinetic energy density $\mathcal{E}$ along 1-pulse convecton branches in a 10 wavelength domain as a function of $\mathrm{Ra}$ for $\mathrm{Ta}=20, \sigma=0.1$ for several different values of $\beta$. For clarity, the periodic branches have been omitted. The meshes used in all cases are identical, although it is to be noted that the critical wavelength at onset varies with $\beta$, implying different domain sizes and hence different element aspect ratios.

\section{DISCUSSION}

In this paper, we have computed for the first time spatially localized convecting states in a rotating fluid layer with no-slip boundary conditions at top and bottom. These states are present for similar values of the Prandtl and Taylor numbers as in the corresponding stress-free case but at larger Rayleigh numbers, and may be stable, at least with respect to two-dimensional perturbations. The no-slip boundary conditions break the conservation of the zonal momentum and change substantially the behavior of the system. In particular, the zonal velocity is now strongly coupled to the poloidal flow and hence varies on the same spatial scale - the zonal velocity is no longer a conserved large scale mode. As a result slanted snaking is absent, and the convectons instead undergo standard homoclinic snaking in a well-defined Rayleigh number interval much as in other problems with no-slip boundary conditions. ${ }^{8,28,38,39}$ In particular, we no longer expect to find convectons below the saddle-node of the periodic states or in the supercritical regime, and no such solutions have been found.

To provide insight into the way the snaking region changes as the conservation law is progressively broken, we developed a parametrized interpolation between stress-free and no-slip boundary conditions that preserves the symmetries of the problem, and used this interpolation to continue known stress-free convectons corresponding to the parameter $\beta=0$ first to nonzero values of $\beta$ and then to $\beta=1$, corresponding to no-slip boundary conditions. Once a $\beta \neq 0$ convecton was found it was used to initialize continuation in the Rayleigh number Ra thereby allowing us to construct the whole convecton branch at that value of $\beta$ and in particular to determine its origin and termination. As part of this study, we found that for larger rotation rates the 1-pulse convectons terminate on a branch of 2-pulse convectons, instead of on periodic states. We attributed this unexpected behavior to the strong longitudinal shear set up across the convecton owing to the no-slip boundaries rotating with angular velocity $\Omega$ and the tendency of the interior of the layer to rotate more slowly owing to the expulsion of zonal velocity gradients from regions of closed contours. This explanation suggests, and detailed computations confirm, that this splitting of convectons that are too broad occurs only for small to moderate values of the parameter $\beta$ and sufficiently rapid rotation. For lower rotation rates, we found instead that the snaking behavior starts out as if it were to slant, but that with increasing Rayleigh number this behavior transitions into standard snaking familiar from systems without a conserved quantity. We have referred to this type of behavior as curved snaking. It reflects a fundamental difference between conserved and nonconserved systems. In the former, the convectons always fill the same fraction of the domain at fixed Rayleigh number regardless of domain size. In the latter, the convectons are independent of the domain size once this is large enough. 
We mention that convecton splitting can take place even in systems with a conserved quantity such as magnetoconvection ${ }^{37}$ while in other systems it occurs as a result of nonperiodic or nonNeumann boundary conditions in the extended direction..$^{40,41}$ More generally, multipulse states are found in the snaking region of 1-pulse states, both in model equations like the Swift-Hohenberg equation ${ }^{42}$ and in convection problems without a conserved quantity. ${ }^{28}$ In these systems, however, the 1-pulse and 2-pulse states remain distinct, and no connections between them are present. Thus, the observation in the present paper of the termination of 1-pulse states on a branch of 2-pulse states is to our knowledge new. In other systems, such as binary fluid convection, ${ }^{7,38}$ the total mass in the heavier and lighter components of the mixture is conserved by the dynamics but for reasons that are not understood this fact exerts almost no effect on the snaking behavior of the associated localized states although it is responsible for the phenomenon of concentration pumping in the horizontal direction, resulting in a change in the width of the snaking region for odd parity convectons relative to that for even parity convectons. ${ }^{7,28}$

Although the mixed boundary conditions used for our homotopic continuation were constructed for mathematical and numerical convenience, they are in fact of the same form as the first order slip boundary condition obtained by Maxwell ${ }^{43}$ for rarefied gas flow; this boundary condition has since been derived from the Boltzmann equation in the kinetic theory of gases. ${ }^{44}$ Thus, our results for intermediate values of $\beta$ may have physical applicability in the theory of gaseous convection.

\section{ACKNOWLEDGMENTS}

This work was supported by the National Science Foundation (NSF) under Grant No. DMS1211953. E.K. wishes to acknowledge support from the Chaire d'Excellence Pierre de Fermat de la région Midi-Pyrénées (France).

${ }^{1}$ H. E. Huppert and D. R. Moore, "Nonlinear double-diffusive convection," J. Fluid Mech. 78, 821-854 (1976).

${ }^{2}$ M. R. E. Proctor and N. O. Weiss, "Magnetoconvection," Rep. Prog. Phys. 45, 1317-1379 (1982).

${ }^{3}$ G. Veronis, "Cellular convection with finite amplitude in a rotating fluid," J. Fluid Mech. 5, 401-435 (1959).

${ }^{4}$ S. Chandrasekhar, Hydrodynamic and Hydromagnetic Stability (Oxford University Press, Oxford, 1961).

${ }^{5}$ K. Ghorayeb and A. Mojtabi, "Double diffusive convection in a vertical rectangular cavity," Phys. Fluids 9, 2339-2348 (1997).

${ }^{6}$ S. Blanchflower, "Magnetohydrodynamic convectons," Phys. Lett. A 261, 74-81 (1999).

${ }^{7}$ O. Batiste, E. Knobloch, A. Alonso, and I. Mercader, "Spatially localized binary-fluid convection," J. Fluid Mech. 560, 149-156 (2006).

${ }^{8}$ A. Bergeon and E. Knobloch, "Spatially localized states in natural doubly diffusive convection," Phys. Fluids 20, 034102 (2008).

${ }^{9}$ P. Assemat, A. Bergeon, and E. Knobloch, "Spatially localized states in Marangoni convection in binary mixtures," Fluid Dyn. Res. 40, 852-876 (2008).

${ }^{10}$ I. Mercader, O. Batiste, A. Alonso, and E. Knobloch, “Travelling convectons in binary fluid convection,” J. Fluid Mech. 722, 240-266 (2013).

${ }^{11}$ J. Burke and E. Knobloch, "Localized states in the generalized Swift-Hohenberg equation," Phys. Rev. E 73, 056211 (2006).

12 J. Burke and E. Knobloch, "Snakes and ladders: Localized states in the Swift-Hohenberg equation," Phys. Lett. A 360, 681-688 (2007)

${ }^{13}$ M. Beck, J. Knobloch, D. J. B. Lloyd, B. Sandstede, and T. Wagenknecht, "Snakes, ladders and isolas of localized patterns," SIAM J. Math. Anal. 41, 936-972 (2009).

${ }^{14}$ D. Avitabile, D. J. B. Lloyd, J. Burke, E. Knobloch, and B. Sandstede, "To snake or not to snake in the planar SwiftHohenberg equation,” SIAM J. Appl. Dyn. Syst. 9, 704-733 (2010).

${ }^{15}$ J. H. P. Dawes, "Localized pattern formation with a large-scale mode: slanted snaking," SIAM J. Appl. Dyn. Syst. 7, 186-206 (2008).

${ }^{16}$ U. Thiele, A. J. Archer, M. J. Robbins, H. Gomez, and E. Knobloch, "Localized states in the conserved Swift-Hohenberg equation with cubic nonlinearity,” Phys. Rev. E 87, 042915 (2013).

${ }^{17}$ D. Lo Jacono, A. Bergeon, and E. Knobloch, "Magnetohydrodynamic convectons,” J. Fluid Mech. 687, 595-605 (2011).

${ }^{18}$ C. Beaume, A. Bergeon, H.-C. Kao, and E. Knobloch, "Convectons in a rotating fluid layer," J. Fluid Mech. 717, 417-448 (2013).

${ }^{19}$ J. H. P. Dawes, "Localized convection cells in the presence of a vertical magnetic field," J. Fluid Mech. 570, 385-406 (2007).

${ }^{20}$ G. Küppers and D. Lortz, "Transition from laminar convection to thermal turbulence in a rotating fluid layer," J. Fluid Mech. 35, 609-620 (1969).

${ }^{21}$ A. Rubio, J. M. Lopez, and F. Marques, “Onset of Küppers-Lortz-like dynamics in finite rotating thermal convection,” J Fluid Mech. 644, 337-357 (2010). 
${ }^{22}$ K. M. S. Bajaj, G. Ahlers, and W. Pesch, "Rayleigh-Bénard convection with rotation at small Prandtl numbers," Phys. Rev. E 65, 056309 (2002).

${ }^{23}$ P. Vorobieff and R. E. Ecke, "Turbulent rotating convection: An experimental study,” J. Fluid Mech. 458, 191-218 (2002).

${ }^{24}$ M. Sprague, K. Julien, E. Knobloch, and J. Werne, "Numerical simulation of an asymptotically reduced system for rotationally constrained convection," J. Fluid Mech. 551, 141-174 (2006).

${ }^{25}$ R. Seydel, Practical Bifurcation and Stability Analysis, Interdisciplinary Applied Mathematics Vol. 5 (Springer Verlag, New York, 2010).

${ }^{26}$ L. S. Tuckerman, "Steady-state solving via Stokes preconditioning: Recursion relations for elliptic operators," in Proceed ings of the 11th International Conference on Numerical Methods in Fluid Dynamics, Lecture Notes in Physics Vol. 323, edited by D. Dwoyer, M. Hussaini, and R. Voigt (Springer, Berlin, 1989), pp. 573-577.

${ }^{27}$ C. K. Mamun and L. S. Tuckerman, "Asymmetry and Hopf bifurcation in spherical Couette flow," Phys. Fluids 7, 80-91 (1995).

${ }^{28}$ C. Beaume, A. Bergeon, and E. Knobloch, "Homoclinic snaking of localized states in doubly diffusive convection," Phys. Fluids 23, 094102 (2011).

${ }^{29}$ A. Bogusławski and S. Kubacki, "An influence matrix technique for multi-domain solution of the Navier-Stokes equations in a vorticity-streamfunction formulation," J. Theor. Appl. Mech. 47, 17-40 (2009).

${ }^{30}$ S. M. Cox and P. C. Matthews, "Instability of rotating convection," J. Fluid Mech. 403, 153-172 (2000).

${ }^{31}$ S. M. Cox and P. C. Matthews, "New instabilities in two-dimensional rotating convection and magnetoconvection," Physica D 149, 210-229 (2001).

${ }^{32}$ N. O. Weiss, "The expulsion of magnetic flux by eddies," Proc. R. Soc. London, Ser. A 293, 310-328 (1966)

${ }^{33}$ P. B. Rhines and W. R. Young, "How rapidly is a passive scalar mixed within closed streamlines?," J. Fluid Mech. 133, 133-145 (1983).

${ }^{34}$ J. Mason and E. Knobloch, "Long dynamo waves," Physica D 205, 100-124 (2005).

${ }^{35}$ J. R. Burke, "Localized states in driven dissipative systems," Ph.D thesis, University of California at Berkeley, 2008.

${ }^{36}$ E. J. Doedel, A. R. Champneys, F. Dercole, T. Fairgrieve, Y. Kuznetsov, B. Oldeman, R. Paffenroth, B. Sandstede, X. Wang, and C. Zhang, AUTO-07P: Continuation and Bifurcation Software for Ordinary Differential Equations (Concordia University, 2008).

${ }^{37}$ D. Lo Jacono, A. Bergeon, and E. Knobloch, "Spatially localized magnetoconvection," Fluid Dyn. Res. 44, 031411 (2012).

${ }^{38}$ D. Lo Jacono, A. Bergeon, and E. Knobloch, "Spatially localized binary fluid convection in a porous medium," Phys. Fluids 22, 073601 (2010).

${ }^{39}$ T. M. Schneider, J. F. Gibson, and J. Burke, "Snakes and ladders: Localized solutions of plane Couette flow," Phys. Rev. Lett. 104, 104501 (2010).

${ }^{40}$ S. M. Houghton and E. Knobloch, "Homoclinic snaking in bounded domains," Phys. Rev. E 80, 026210 (2009).

${ }^{41}$ C. Beaume, A. Bergeon, and E. Knobloch, "Convectons and secondary snaking in three-dimensional natural doubly diffusive convection," Phys. Fluids 25, 024105 (2013).

${ }^{42}$ J. Burke and E. Knobloch, "Multipulse states in the Swift-Hohenberg equation,” Discrete Contin. Dyn. Syst., Supplement Volume, pp. 109-117 (2009)

${ }^{43}$ E. H. Kennard, Kinetic Theory of Gases (McGraw-Hill, New York, 1938)

${ }^{44}$ S. Shen, G. Chen, R. Crone, and M. Anaya-Dufresne, "A kinetic-theory based first order slip boundary condition for gas flow," Phys. Fluids 19, 086101 (2007). 